\title{
Creation of Two Charginos and Neutralino Via Different Propagators
}

\author{
Zainab.A.H Asmaa.A. A. M.M. Ahmed* \\ *Physics Department, Helwan University Cairo, Egypt
}

\begin{abstract} graphs which taken by the sorts of the propagators. recognized as:

$$
\begin{aligned}
& e^{-}\left(P_{1}\right)+e^{+}\left(P_{3}\right) \rightarrow Z^{o}\left(P_{2}+P_{4}\right) \rightarrow \tilde{\chi}_{l}^{-}\left(P_{2}\right)+\tilde{\chi}_{j}^{+}\left(P_{4}\right) \text { in group I. } \\
& e^{-}\left(P_{1}\right)+e^{+}\left(P_{3}\right) \rightarrow h^{o}\left(P_{2}+P_{4}\right) \rightarrow \tilde{\chi}_{l}^{-}\left(P_{2}\right)+\tilde{\chi}_{j}^{+}\left(P_{4}\right) \text { in group II. } \\
& e^{-}\left(P_{1}\right)+e^{+}\left(P_{3}\right) \rightarrow H^{o}\left(P_{2}+P_{4}\right) \rightarrow \tilde{\chi}_{l}^{-}\left(P_{2}\right)+\tilde{\chi}_{j}^{+}\left(P_{4}\right) \text { in group III }
\end{aligned}
$$
\end{abstract}

We investigated the creation of two Charginos $\left(\tilde{\chi}^{ \pm}\right)$and neutralino $\left(\tilde{\chi}^{\mathrm{o}}\right)$ owing to electron-positron annihilation via the process $e^{-}\left(p_{1}\right)+e^{+}\left(p_{3}\right) \rightarrow \tilde{\chi}_{i}^{-}\left(p_{2}\right)+\tilde{\chi}_{j}^{+}\left(p_{4}\right)+\tilde{\chi}_{\ell}^{\circ}\left(p_{5}\right)$ and estimated the cross section for this interaction in the Minimal Supersymmetric Standard Model (MSSM). There are three gatherings of Feynman

Group (I) when $\tilde{\chi}^{\mathrm{o}}$ and $\mathrm{Z}^{\mathrm{o}}$ boson are propagators, Group (II) when $\tilde{\chi}^{\mathrm{o}}$ and $\mathrm{h}^{\mathrm{o}}$ (lighter Higgs boson) are propagators and Group (III) when $\tilde{\chi}^{0}$ and $\mathrm{H}^{\mathrm{o}}$ (heavier Higgs boson) are propagators, where $i, j=1,2$ and $\ell=$ $1,2,3,4$. here are (192) various potential circumstances from Feynman graph. Over a range of center of mass energy $\mathrm{S}(\mathrm{Gev})$, the cross-sections ( $\mathrm{pb})$ are determined based on (MSSM), the mechanisms of the process can be

At $\mathrm{S}$ interval (1000-2100) Gev, the best value of $\sigma$ is ( 0.072$) \mathrm{Pb}$ in-group (I). When masses of Charginos are $\mathrm{m}_{\tilde{\chi}_{i}^{-}}=700 \mathrm{GeV}, \mathrm{m}_{\tilde{\chi}_{j}^{+}}=700 \mathrm{GeV}$ and mass of neutralino is $\mathrm{m}_{\tilde{\chi}_{\ell}^{0}}=800 \mathrm{GeV}$

Keywords: Chargino, neutralino and Higgs boson

DOI: $10.7176 /$ APTA/84-02

Publication date:August $31^{\text {st }} 2021$

\section{Introduction}

Understanding the mechanism that causes breakdown of the electroweak symmetry is one of particle physics "most important unsolved challenges". the SU (2) $\times$ U (1) group is expected to be spontaneously broken in the Standard Model (SM) [1] and $W^{ \pm}$and $Z^{0}$ bosons acquire their masses through Higgs mechanism. $M_{W^{ \pm}}=80 \mathrm{Gev}, M_{z}=$ $91 \mathrm{Gev}$

Only Higgs' couplings with matter and gauge particles are expected in the SM, but its mass is not well known theoretically [1]. The standard model predicts a single neutral Higgs boson $H^{\circ}$ that is spin-zero with the $J^{P C}=0^{++}$ and is even under charge conjugation and parity (CP) [2]. Despite the fact that the mass of $H^{\circ}$ is a free parameter, The theory's self-consistency necessitates a mass of less than $1 \mathrm{TeV}[3,4]$.

Supersymmetry (SUSY) is a symmetry that connects particles with different spins by combining them into a super field with fields that differ by one-half unit of spin [4].

The theoretical foundation is the Minimal Supersymmetric Standard Model (MSSM), which contains an extended scalar sector with two doublets of Higgs fields $\Phi_{1}$ and $\Phi_{2}$ where $\Phi_{1}\left(v_{1}\right)$ and $\Phi_{2}\left(v_{2}\right)$ are needed to give masses to up- and down-type fermions [5]. In contrast to the SM, where the Higgs Mechanism can generate both $\mathrm{u}$ (up) and d (down) type masses with just one Higgs doublet [6].

The two-doublet Higgs model (2DHM) has five Higgs particles: two neutral CP-even scalar $J^{P C}=0^{++}(\mathrm{h}$ for the lighter one and $\mathrm{H}$ for the heavier one), one neutral CP-odd pseudo-scalar $J^{P C}=0^{-+}(\mathrm{A})$, and two charged $\left(H^{ \pm}\right)$ bosons $[7,8]$. The lighter $\mathrm{CP}$-even scalar $(\mathrm{h})$ is the measured boson at $125 \mathrm{GeV}[6]$.

The CP-odd Higgs boson (A) does not couple to $W^{+} W^{-}$and $\mathrm{ZZ}$ pairs at tree level in contrast to the CP-even Higgs bosons ( $h$ and $\mathrm{H}$ ), and does not couple to other two physical Higgs bosons in cubic interactions $\mathrm{n}$. The coupling of (A) to quarks and leptons are proportional to their masses [9]

Six parameters can describe the Higgs sector of a $2 \mathrm{HDM}$ : four Higgs boson masses $\left(\mathrm{m}_{\mathrm{h}}, \mathrm{m}_{\mathrm{H}}, \mathrm{m}_{\mathrm{A}}\right.$ and $\left.\mathrm{m}_{\mathrm{H}^{ \pm}}\right)$, the ratio of the vacuum expectation values of the two higgs doublets $\left(\tan \beta=v_{2} / v_{1}\right)$ and the mixing angle $\alpha$ of the two neutral CP-even Higgs states [6]

The MSSM model has two Higgs doublets and additional constraints [10,11].

$m_{\mathrm{A}}^{2}+M_{Z}^{2}=m_{\mathrm{H}}^{2}+m_{\mathrm{h}}^{2}$,

$\mathrm{m}_{\mathrm{H}^{ \pm}}^{2}=\mathrm{M}_{\mathrm{A}}^{2}+\mathrm{M}_{\mathrm{W}}^{2}$

$\mathrm{m}_{\mathrm{h}} \leq \mathrm{m}_{\mathrm{Z}}|\cos 2 \beta| \leq \mathrm{m}_{\mathrm{Z}}$ according to SUSY model

the lightest Higgs boson mass in the MSSM $m_{h}$ reads

$\mathrm{m}_{\mathrm{h}}^{2}=\mathrm{m}_{\mathrm{Z}}^{2} \cos ^{2} 2 \beta+\frac{3 \mathrm{~g}^{2} \mathrm{~m}_{\mathrm{t}}^{4}}{16 \pi^{2} \mathrm{M}_{\mathrm{W}}^{2}} \log \frac{\widetilde{\mathrm{m}}_{\mathrm{t}_{1}}^{2} \widetilde{\mathrm{m}}_{\mathrm{t}_{2}}^{2}}{\mathrm{~m}_{\mathrm{t}}^{4}}$ 
Which leads to about $40 \mathrm{GeV}$ increase [12], where $m_{t}$ mass of the top quark

From these constraints, it also follows that

$\mathrm{m}_{\mathrm{h}}<\mathrm{m}_{\mathrm{A}}<\mathrm{m}_{\mathrm{H}}$

$\mathrm{m}_{\mathrm{H}, \mathrm{h}}^{2}=\frac{1}{2}\left[\mathrm{M}_{\mathrm{A}}^{2}+\mathrm{M}_{\mathrm{Z}}^{2} \pm \sqrt{\left(\mathrm{M}_{\mathrm{A}}^{2}+\mathrm{M}_{\mathrm{Z}}^{2}\right)^{2}-4 \mathrm{M}_{\mathrm{Z}}^{2} \mathrm{M}_{\mathrm{A}}^{2} \cos ^{2} 2 \beta}\right]$

Where the masses $m_{H}, m_{h}, m_{A}, m_{H^{ \pm}}$of the Higgs particles $H^{\circ}, h^{\circ}, A^{\circ}, H^{ \pm}$respectively.

The two angles $\beta$ and $\alpha$ fixed in terms of the Higgs boson masses [13].

$\cos 2 \alpha=-\cos 2 \beta\left[\left(\mathrm{m}_{\mathrm{A}}^{2}-\mathrm{M}_{\mathrm{z}}^{2}\right) /\left(\mathrm{m}_{\mathrm{h}}^{2}-\mathrm{M}_{\mathrm{z}}^{2}\right)\right]$.

$\sin 2 \alpha=-\sin 2 \beta\left[\left(m_{\mathrm{h}}^{2}+\mathrm{m}_{2}^{2}\right) /\left(\mathrm{m}_{\mathrm{h}}^{2}-\mathrm{m}_{\mathrm{H}}^{2}\right)\right]$.

$\tan 2 \alpha=\tan 2 \beta\left[\left(\mathrm{m}_{\mathrm{A}}^{2}-\mathrm{M}_{\mathrm{z}}^{2}\right) /\left(\mathrm{m}_{\mathrm{A}}^{2}-\mathrm{M}_{\mathrm{z}}^{2}\right)\right]$.

The angel $\alpha$ lie in the interval $-\pi / 2 \leq \alpha \leq 0$. And the angel $\beta$ can be taken to lie in the interval $0 \leq \beta \leq$ $\pi / 2 \cdot[14]$

In particle physics, slepton is a Superpartner of a lepton that described by Supersymmetry. It has the same flavor and electric charge alike leptons and their spin is zero. For example, selectron $\tilde{e}_{h}$ is superpartner of electron [4].

In the minimal supersymmetric standard model (MSSM), the superpartners of the electroweak gauge $\left(\mathrm{Z}^{\circ}, \mathrm{W}^{ \pm}\right)$ and Higgs bosons mix to form electroweakinos. These consist of four Majorana fermions (neutralinos $\tilde{\chi}_{\mathrm{i}}^{\circ}$, with $i=1,2,3,4)$, and two Dirac fermions (charginos $\tilde{\chi}_{\mathrm{i}}^{ \pm}$, with $\left.i=1,2\right)[15]$

\subsection{Cross-sections meaning}

The term "cross section" its technical meaning is very different from the common usage. In everyday speech, "cross section" refers to a slice of an object. A particle physicist might use the word this way, but more often it is used to mean the probability that two particles will collide and react in a certain way. For instance, when CMS physicists measure the "proton-proton to top-antitop" cross section, they are counting how many top-antitop pairs were created when a given number of protons were fired at each other. But why use "cross section" when alternatives like "probability" and "reaction rate" exist? Cross section is independent of the intensity and focus of the particle beams, so cross section numbers measured at one accelerator can be directly compared with numbers measured at another, regardless of how powerful the accelerators are.

\subsection{Scattering cross-sections}

Almost everything we know about nuclear and atomic physics has been discovered by scattering experiments, e.g., Rutherford's discovery of the nucleus, the discovery of sub-atomic particles (such as quarks), etc. In low energy physics, scattering phenomena provide the standard tool to explore solid state systems, e.g., neutron, electron, $\mathrm{x}-$ ray scattering, etc. As a general topic, it therefore remains central to any advanced course on quantum mechanic

A scattering cross-section, $\sigma$, is a quantity proportional to the rate at which a particular radiation-target interaction occurs. More specifically, if the incoming radiation is considered as being composed of quanta or 'particles' (for example, photons or neutrons), a cross-section is a scattering rate (number of scattering events per unit time) per unit incident radiation flux, where the latter is the number of incident particles striking the target surface per unit time per unit area. In cases where the radiation is being treated as a continuous classical wave, as in the case of long-wavelength electromagnetic radiation, scattering cross-sections are determined by dividing the power of the scattered wave by the intensity of the incident wave. Dimensionally, a cross-section represents an area, with the basic unit being the barn, which represents an area of 10-28 $\mathrm{m}^{2}$. A scattering cross-section should not be interpreted as a true geometric cross-sectional area, but as an effective area that is proportional to the probability of interaction between the radiation and target.

In a real scattering experiment, information about the scatterer can be figured out from the different rates of scattering to different angles. Detectors are placed at various angles $(\theta, \phi)$ Of course, a physical detector collects scattered particles over some nonzero solid angle. The usual notation for infinitesimal solid angle is $d \Omega=$ $\sin \theta d \theta d \phi$. The full solid angle (all possible scatterings) is $\int d \Omega=4 \pi$ the area of a sphere of unit radius.

The differential cross section, written $d \sigma / d \Omega$ is the fraction of the total number of scattered particles that come out in the solid angle $d \Omega$, so the rate of particle scattering to this detector is $n d \sigma / d \Omega$, with $\mathrm{n}$ the beam intensity as defined above.

From the differential, we can obtain the total cross section by integrating over all solid angles

The cross section depends sensitively on energy of incoming particles

$$
\sigma=\int \frac{d \sigma}{d \Omega} d \Omega=\int_{0}^{2 \pi} d \varphi \int_{0}^{\pi} d \theta \sin \theta \frac{d \sigma}{d \Omega}
$$

\subsection{Feynman diagram}

Richard Feynman developed a technique referred to as Feynman diagrams. The essence of these diagrams is that 
they portrayed quantum events as trajectories. For example, along the time axis an electron and positron (antielectron) particle annihilate each other producing a virtual photon that becomes a quark-antiquark pair.

Feynman diagrams are in common use in particle physics. The value of these diagrams is to facilitate the calculation of interactions between particles. The introduction of these diagrams contributed to the theory of QED, first introduced by Dirac. Along with two others, Richard Feynman was awarded the Nobel Prize in 1965 for work in electrodynamics and consequences for the physics of elementary particles.

These diagrams are one of the fundamental tools used to make precise calculations for the probability of occurrence of any process by physicists. Different diagrams can represent a single interaction process, and the contribution from each diagram is taken into consideration while calculating this probability. Although the mathematical expressions involved in calculating these probabilities are quite complex, a lot simpler as compared to other techniques.

Although the American theoretical physicist Richard Feynman first introduced these diagrams only as a bookkeeping device for simplifying lengthy calculations in the area of quantum electrodynamics, these diagrams have come a long way now. Even David Kaiser once quoted, "Since the middle of the 20th century, theoretical physicists have increasingly turned to this tool to help them undertake critical calculations. Feynman diagrams have revolutionized nearly every aspect of theoretical physics." Undoubtedly, these diagrams are one of Feynman's finest contributions ever made to the Physics fraternity.

\subsection{Properties of Charginos:}

- Charginos are composed of Winos $\left(W^{+}, W^{-}\right)$and Higgsinos $\left(H^{+}, H^{-}\right)[16,17]$.

- In nature neutralino dark matter observed experimentally either indirectly by using $\gamma$ ray and neutrino telescopes or directly by using an array of semiconductor detectors and through experiments such as Cryogenic dark matter search (CDMS) it is a series of experiments designed to detect particle dark matter directly in the form of WIMPs [18, 19].

- The heavier neutralinos typically decay through a neutral $\mathrm{Z}$ boson to a lighter neutralino or through a charged W boson to a light chargino [20]

- $\quad$ Produced in pairs via s-channel $\gamma / Z$ exchange $[21,22]$.

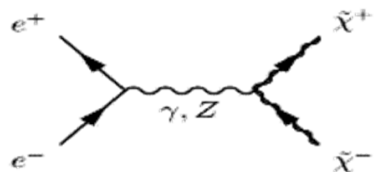

Fig. (1): Feynman diagrams for chargino pair production via s-channel

- The lightest chargino $\tilde{\chi}_{1}^{ \pm}$is slightly heavier than the lightest neutralino $\tilde{\chi}_{1}^{\circ}$.

- The expression of the mean lifetime $\left(\tau_{\widetilde{\chi}_{1}^{ \pm}}\right)$of $\tilde{\chi}_{1}^{ \pm}$in terms of $\Delta m_{\widetilde{\chi}_{1}}$ and expected to be typically a fraction of a nanosecond. Lifetime of charginos between 0.1 and $10 \mathrm{~ns}$ [23].

- The charginos decay into the lightest neutralino $\tilde{\chi}_{1}^{\circ}$, which is taken to be stable, and a pair of fermions $(f)$ which are quarks and antiquarks or leptons and neutrinos:[24]

- $\quad \tilde{\chi}_{1}^{ \pm} \rightarrow \tilde{\chi}_{1}^{\circ}+f \bar{f}$

- The lightest chargino mass greater than $103.5 \mathrm{GeV}[25]$

- The second chargino $\tilde{\chi}_{2}^{ \pm}$is generally expected to be significantly heavier than the first state.[24]

- There are three variables or soft terms $\left(M_{2}, \mu\right.$ and $\left.\tan \beta\right)$ in chargino mass matrix and four variables or soft terms $\left(M_{1}, M_{2}, \mu\right.$ and $\left.\tan \beta\right)$ in neutralino mass matrix [26]

Where:

$M_{1}$ is the soft-breaking bino mass

$M_{2}$ is the soft-breaking wino mass

$\mu$ is the Higgsino superpotential mass parameter

$\tan \beta$ is the ratio of the two Higgs vacuum expectation values

$$
\begin{gathered}
m_{\widetilde{\chi}_{1}^{ \pm}} \simeq m_{\widetilde{\chi}_{2}^{\circ}} \simeq 2 m_{\widetilde{\chi}_{1}^{\circ}} \\
m_{\widetilde{\chi}_{2}^{ \pm}} \simeq m_{\widetilde{\chi}_{3}^{\circ}} \simeq m_{\widetilde{\chi}_{4}^{\circ}} \simeq|\mu|
\end{gathered}
$$

$M_{1}=\frac{5}{3} \tan \theta_{w}^{2} M_{2}$ The relation in grand unification theory GUT between $M_{1}$ and $M_{2}$ :

Where $\theta_{w}$ is the Weinberg angle and

$$
\cos \theta_{w}=\frac{M_{w}}{M_{z}}
$$

2. Calculation Cross sections in $(\mathrm{Pb})$ :

3-body final states with momenta $\mathrm{p}_{2}, \mathrm{p}_{4}, \mathrm{p}_{5}$ produced and their masses $\mathrm{m}_{2}, \mathrm{~m}_{4}, \mathrm{~m}_{5}$ and the initial states have 
momenta $\mathrm{p}_{1}, \mathrm{p}_{3}$ and their masses $\mathrm{m}_{1}, \mathrm{~m}_{3}$.

$$
\begin{aligned}
\mathrm{p}_{1}+\mathrm{p}_{3} & =\mathrm{p}_{2}+\mathrm{p}_{4}+\mathrm{p}_{5} \\
\mathrm{~s} & =\sigma+\mathrm{p}_{5}
\end{aligned}
$$

The cross section $(\sigma)$ for the process $\mathrm{e}^{-}\left(\mathrm{p}_{1}\right)+\mathrm{e}^{+}\left(\mathrm{p}_{3}\right) \rightarrow \tilde{\chi}_{\mathrm{i}}^{-}\left(\mathrm{p}_{2}\right)+\tilde{\chi}_{\mathrm{j}}^{+}\left(\mathrm{p}_{4}\right)+\tilde{\chi}_{\ell}^{\circ}\left(\mathrm{p}_{5}\right)$ can be written in the form

$$
\sigma=\int \pi^{2}|\mathrm{M}|^{2} \frac{\mathrm{dxdy} \mathrm{d \sigma ^{2 }}}{\Lambda\left(\mathrm{S}, \mathrm{m}_{1}, \mathrm{~m}_{3}\right) \Lambda\left(\mathrm{S}, \sigma, \mathrm{m}_{5}\right)}
$$

Where $\mathrm{M}$ is the matrix element, by applying Feynman rules we can write the M-matrix for the Feynman diagram and the trace thermos used to calculate the square matrix $\left(|M|^{2}\right)$, the integration performed using a simple approximation obtained by an improved Weizsacker-Williamson procedure $[27,28]$. Where:

$\Lambda(\mathrm{x}, \mathrm{y}, \mathrm{z})=\left[\mathrm{x}^{4}+\mathrm{y}^{4}+\mathrm{z}^{4}-2 \mathrm{x}^{2} \mathrm{y}^{2}-2 \mathrm{x}^{2} \mathrm{z}^{2}-2 \mathrm{y}^{2} \mathrm{z}^{2}\right]^{1 / 2}$

Then, by using Mathematica program the integration simplifying and the limit of integration are

$\mathrm{x}_{ \pm}=\frac{1}{4 \mathrm{~S}^{2}}\left[\left(\mathrm{~S}^{2}+\mathrm{m}_{1}^{2}-\mathrm{m}_{3}^{2}\right)\left(\mathrm{S}^{2}-\sigma^{2}+\mathrm{m}_{5}^{2}\right) \pm \Lambda\left(\mathrm{S}, \mathrm{m}_{1}, \mathrm{~m}_{3}\right) \Lambda\left(\mathrm{S}, \sigma, \mathrm{m}_{5}\right)\right]$

$\mathrm{y}_{ \pm}=\frac{1}{4 \sigma^{2}}\left[\left(\sigma^{2}+\mathrm{m}_{2}^{2}-\mathrm{m}_{4}^{2}\right)\left(\mathrm{S}^{2}-\sigma^{2}+\mathrm{m}_{5}^{2}\right) \pm \Lambda\left(\sigma, \mathrm{m}_{2}, \mathrm{~m}_{4}\right) \Lambda\left(\mathrm{S}, \sigma, \mathrm{m}_{5}\right)\right]$

$\left(\mathrm{m}_{2}+\mathrm{m}_{4}\right)^{2} \leq \sigma^{2} \leq\left(\mathrm{S}^{2}-\mathrm{m}_{5}^{2}\right)^{2}$

The cross sections calculated and the results graphed and tabled.

In all our calculations, we assume the following values for vector-boson masses [10, 29] suggested:

$\mathrm{M}_{\mathrm{W}}=80 \mathrm{GeV}$

$\mathrm{M}_{\mathrm{Z}}=91 \mathrm{GeV}$

$\mathrm{M}_{\mathrm{h}^{0}}=125 \mathrm{GeV}$ (mass of $\mathrm{h}^{\mathrm{o}}$ propagator)

$\mathrm{M}_{\mathrm{H}^{0}}=140 \mathrm{GeV}$ (mass of $\mathrm{H}^{\mathrm{O}}$ propagator)

$\mathrm{M}_{\tilde{\chi}^{\mathrm{o}}}=300 \mathrm{GeV}$ (mass of $\tilde{\chi}^{\mathrm{o}}$ propagator)

$\mathrm{m}_{\tilde{\chi}_{(i)}^{-}}=(600,700) \mathrm{GeV}, \quad \mathrm{m}_{\tilde{\chi}_{(j)}^{+}}=(600,700) \mathrm{GeV}(i, j=1,2)$

$\mathrm{m}_{\tilde{\chi}_{1}^{0}}=300 \mathrm{GeV}, \mathrm{m}_{\tilde{\chi}_{2}^{0}}=600 \mathrm{GeV}, \mathrm{m}_{\tilde{\chi}_{3}^{0}}=700 \mathrm{GeV}, \mathrm{m}_{\tilde{\chi}_{4}^{0}}=800 \mathrm{GeV}$

\section{Feynman Diagram and M-Matrix for the process, when $\widetilde{\chi}^{0}$ and $\mathrm{Z}^{\mathrm{o}}$ are the propagators Group (I):}

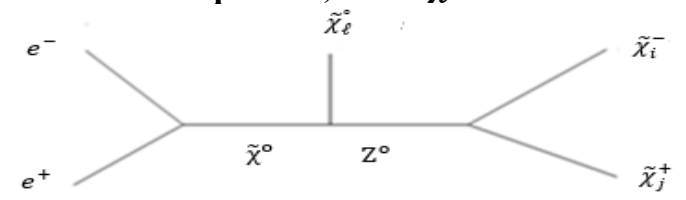

Fig.2: Feynman diagram for the process $e^{-}\left(p_{1}\right)+e^{+}\left(p_{3}\right) \rightarrow \tilde{\chi}_{i}^{-}\left(p_{2}\right)+\tilde{\chi}_{j}^{+}\left(p_{4}\right)+\tilde{\chi}_{\ell}^{\circ}\left(p_{5}\right)$ via $\tilde{\chi}^{\mathrm{o}}$ and $\mathrm{Z}^{\mathrm{o}}$ propagators, there are (1-64) diagrams.

-For group (I) there are 64 situations from (1-64) and the Matrix element is:

$$
\begin{gathered}
M_{(1-64)}=\frac{g^{3} m_{e}\left(\cos ^{2} \theta_{w}-\sin ^{2} \theta_{w}\right)}{8 m_{w} \cos ^{2} \theta_{w}} U_{e^{-}}\left(P_{1}\right) \bar{V}_{e^{+}}\left(P_{3}\right) \gamma_{5} \frac{s+\mathrm{m}_{\tilde{\chi}^{\mathrm{o}}}}{s^{2}-m_{\tilde{\chi}^{\mathrm{o}}}^{2}} \gamma_{v} \overline{\mathrm{U}}_{\tilde{\chi}^{0}}\left(\mathrm{P}_{5}\right)\left(\sigma^{2}-\mathrm{m}_{\mathrm{Z}}^{2}\right)^{-1}\left(\mathrm{P}_{2}\right. \\
\left.+\mathrm{P}_{4}\right)_{\mathrm{K}} \overline{\mathrm{U}}_{\tilde{\chi}^{-}}\left(\mathrm{P}_{2}\right) \overline{\mathrm{U}}_{\tilde{\chi}^{+}}\left(\mathrm{P}_{4}\right)
\end{gathered}
$$

Where:

$g:$ The gauge coupling constants of $\mathrm{SU}(2)_{\mathrm{L}}$

$m_{e}$ : The mass of electron

\subsection{Calculation Cross Sections in (Pb) for Group (I):}

By applying Feynman rules and using equation (3) and Mathematica program, the cross sections calculated as a function of center of mass energy for the Feynman diagram of fig. (2). the results given in figs.3 (a-d) by interchanging the mass of charginos $\left(\mathrm{m}_{\tilde{\chi}_{i}^{-}}, \mathrm{m}_{\tilde{\chi}_{j}^{+}}\right)$at different mass of Neutralino $\mathrm{m}_{\tilde{\chi}_{\ell}^{\circ}}$ for the process $\mathrm{e}^{-}\left(\mathrm{p}_{1}\right)+$ $\mathrm{e}^{+}\left(\mathrm{p}_{3}\right) \rightarrow \tilde{\chi}_{\mathrm{i}}^{-}\left(\mathrm{p}_{2}\right)+\tilde{\chi}_{\mathrm{j}}^{+}\left(\mathrm{p}_{4}\right)+\tilde{\chi}_{\ell}^{\circ}\left(\mathrm{p}_{5}\right)$

$$
\begin{aligned}
& \mathrm{m}_{\left(\tilde{x}_{i}^{-} \cdot \tilde{\chi}_{j}^{+}\right)} \rightarrow \mathrm{m}_{(600 ، 600)} \rightarrow \mathrm{m}_{11} \text { (blue) } \\
& \mathrm{m}_{\left(\tilde{x}_{\mathrm{i}}^{-} \cdot \tilde{x}_{j}^{+}\right)} \rightarrow \mathrm{m}_{(600 ، 700)} \rightarrow \mathrm{m}_{12} \text { (green) } \\
& \begin{aligned}
& \mathrm{m}_{\left(\tilde{x}_{i}^{-} \cdot \tilde{x}_{j}^{+}\right)} \rightarrow \mathrm{m}_{(700 ، 700)} \rightarrow \mathrm{m}_{22}(\text { red }) \\
& \mathrm{m}_{\left(\tilde{x}_{i}^{-} \cdot \tilde{x}_{j}^{+}\right)} \rightarrow \mathrm{m}_{(700 ، 600)} \rightarrow \mathrm{m}_{21}(\text { pink })
\end{aligned}
\end{aligned}
$$




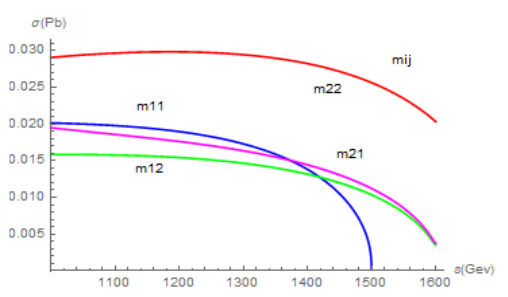

Fig. (3.a) $m_{\tilde{\chi}_{1}^{0}}=300 \mathrm{GeV}$

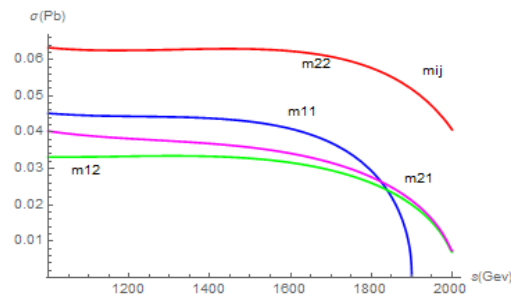

Fig. (3.c) $\mathbf{m}_{\tilde{\chi}_{3}^{0}}=700 \mathrm{GeV}$

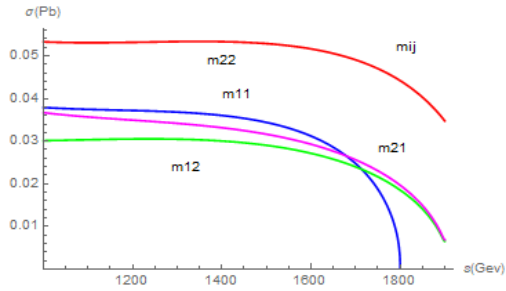

Fig. (3.b) $m_{\tilde{\chi}_{2}^{0}}=600 \mathrm{GeV}$

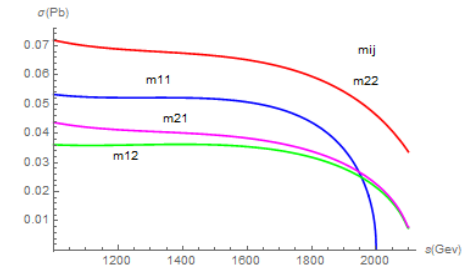

Fig. (3.d) $\mathbf{m}_{\tilde{\chi}_{4}^{0}}=800 \mathbf{~ G e V}$

Fig. 3(a-d): The cross sections for the process $e^{-}\left(p_{1}\right)+e^{+}\left(p_{3}\right) \rightarrow \tilde{\chi}_{i}^{-}\left(p_{2}\right)+\tilde{\chi}_{j}^{+}\left(p_{4}\right)+\tilde{\chi}_{\ell}^{\circ}\left(p_{5}\right)$ as a function of center of mass energy via $\tilde{\chi}^{0}$ and $Z^{0}$ propagators by interchanging the mass of charginos $\left(\mathrm{m}_{\tilde{\chi}_{i}^{-}}, \mathrm{m}_{\tilde{\chi}_{j}^{+}}\right)$at different mass of Neutralino $\mathrm{m}_{\tilde{\chi}_{\ell}^{\circ}}$

Table (1): cross sections for the process $\mathrm{e}^{-}\left(\mathrm{P}_{1}\right)+\mathrm{e}^{+}\left(\mathrm{P}_{3}\right) \rightarrow \mathrm{Z}^{\mathrm{o}}\left(\mathrm{P}_{2}+\mathrm{P}_{4}\right) \rightarrow \tilde{\chi}_{i}^{-}\left(p_{2}\right)+\tilde{\chi}_{j}^{+}\left(p_{4}\right)$ via $\tilde{\chi}^{0}$ and $\mathrm{Z}^{0}$ propagators by interchanging the mass of charginos $\left(\mathrm{m}_{\tilde{\chi}_{\mathrm{i}}^{-}}, \mathrm{m}_{\tilde{\chi}_{j}^{+}}\right)$at different mass of Neutralino $\mathrm{m}_{\tilde{\chi}_{\ell}^{\circ}}$

\begin{tabular}{|c|c|c|c|c|c|c|c|c|}
\hline \multicolumn{9}{|c|}{$\mathbf{e}^{-}\left(\mathbf{P}_{1}\right)+\mathbf{e}^{+}\left(\mathbf{P}_{3}\right) \rightarrow \mathbf{Z}^{\mathbf{o}}\left(\mathbf{P}_{2}+\mathbf{P}_{4}\right) \rightarrow \widetilde{\chi}_{i}^{-}\left(\boldsymbol{p}_{2}\right)+\widetilde{\chi}_{j}^{+}\left(\boldsymbol{p}_{4}\right)$} \\
\hline \multirow{3}{*}{$\mathbf{m}_{\tilde{\chi}_{i}^{-}}-\mathbf{m}_{\tilde{\chi}_{j}^{+}}$} & \multirow{2}{*}{\multicolumn{2}{|c|}{$\begin{array}{l}\tilde{\chi}_{1}^{0}=300 \\
\text { Fig. (3.a) }\end{array}$}} & \multirow{2}{*}{\multicolumn{2}{|c|}{$\begin{array}{l}\widetilde{\chi}_{2}^{0}=600 \\
\text { Fig. (3.b) }\end{array}$}} & \multirow{2}{*}{\multicolumn{2}{|c|}{$\begin{array}{l}\widetilde{\chi}_{3}^{0}=700 \\
\text { Fig. (3.c) }\end{array}$}} & \multicolumn{2}{|c|}{$\widetilde{\chi}_{4}^{0}=\mathbf{8 0 0}$} \\
\hline & & & & & & & Fig. (3. & \\
\hline & S(Gev) & $\sigma(\mathbf{P b})$ & S(Gev) & $\sigma(\mathbf{P b})$ & S(Gev) & $\sigma(\mathbf{P b})$ & S(Gev) & $\sigma(\mathbf{P b})$ \\
\hline 600,600 & 1014 & 0.020 & 1037 & 0.038 & 1030 & 0.045 & 1025 & 0.053 \\
\hline 700,700 & 1202 & 0.030 & 1350 & 0.053 & 1041 & 0.063 & 1015 & 0.072 \\
\hline 600,700 & 1026 & 0.015 & 1230 & 0.030 & 1230 & 0.033 & 1336 & 0.036 \\
\hline 700,600 & 1021 & 0.019 & 959 & 0.037 & 948 & 0.041 & 1064 & 0.042 \\
\hline
\end{tabular}

\subsection{Results for Group (I):}

After studding, the Feynman rules and calculate the cross sections $(\sigma)$ as a function of center of mass energy (S) for the process $e^{-}\left(p_{1}\right)+e^{+}\left(p_{3}\right) \rightarrow \tilde{\chi}_{i}^{-}\left(p_{2}\right)+\tilde{\chi}_{j}^{+}\left(p_{4}\right)+\tilde{\chi}_{\ell}^{\circ}\left(p_{5}\right)$ via $\tilde{\chi}^{0}$ and $\mathrm{Z}^{\mathrm{o}}$ propagators. In figs.3 (a-d) we found that, At $\mathrm{S}$ increase from 1000 to 2100 , we have different maximum values for the cross-sections at different values of Chargino mass $\left(\mathrm{m}_{\tilde{\chi}_{\mathrm{i}}^{-}}, \mathrm{m}_{\tilde{\chi}_{\mathrm{j}}^{+}}\right)$and different value of neutralino mass $\mathrm{m}_{\tilde{\chi}_{\ell}^{0}}$. From table (1) the best value of $\sigma$ is (0.072) Pb when masses of Charginos are $\mathrm{m}_{\tilde{\chi}_{\mathrm{i}}^{-}}=700 \mathrm{GeV}, \mathrm{m}_{\tilde{\chi}_{\mathrm{j}}^{+}}=700 \mathrm{GeV}$ and $\mathrm{m}_{\tilde{\chi}_{\ell}^{0}}=800 \mathrm{GeV}$

\section{Feynman Diagram and M-Matrix for the process, when $\tilde{\chi}^{0}$ and $h^{0}$ are the propagators Group (II):}

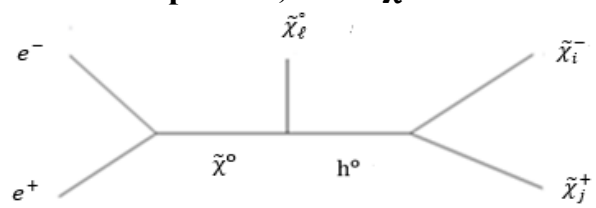

Fig. (4): Feynman diagram for the process $e^{-}\left(p_{1}\right)+e^{+}\left(p_{3}\right) \rightarrow \tilde{\chi}_{i}^{-}\left(p_{2}\right)+\tilde{\chi}_{j}^{+}\left(p_{4}\right)+\tilde{\chi}_{\ell}^{\circ}\left(p_{5}\right)$ via $\tilde{\chi}^{\mathrm{o}}$ and $\mathrm{h}^{\mathrm{o}}$ there are (65-128) diagrams.

- For group (II) there are 64 situations from $(65-128)$ and the Matrix element is:

$$
M_{(65-128)}=\frac{-i g^{2} m_{e}}{4 \sqrt{2} m_{w} \sin \theta_{w}} \mathrm{U}_{\mathrm{e}^{-}}\left(\mathrm{P}_{1}\right) \overline{\mathrm{V}}_{\mathrm{e}^{+}}\left(\mathrm{P}_{3}\right) \gamma_{5} \frac{s+\mathrm{m}_{\chi}}{s^{2}-m_{\chi}^{2}} \overline{\mathrm{U}}_{\tilde{\chi}^{0}}\left(\mathrm{P}_{5}\right) \mathrm{C}\left(\sigma^{2}-\mathrm{m}_{\mathrm{H}}^{2}\right)^{-1} B \overline{\mathrm{U}}_{\tilde{\chi}^{-}}\left(\mathrm{P}_{2}\right) \overline{\mathrm{U}}_{\tilde{\chi}^{+}}\left(\mathrm{P}_{4}\right)
$$

Where: 


$$
\begin{aligned}
& C=\frac{1}{2 \sin \theta_{w}}\left(Z_{j 2}-\tan \theta_{\mathrm{w}} Z_{j 1}\right)\left(e_{k} Z_{i 3}+d_{k} Z_{i 4}\right) \\
& e_{k}=\left(\cos \alpha,-\sin \alpha, \sin \beta_{\circ}\right) \\
& d_{k}=\left(-\sin \alpha, \cos \alpha, \cos \beta_{\circ}\right)
\end{aligned}
$$

$\mathrm{Z}$ is the $(4 \times 4)$ matrices diagonalizing of the neutralino mass

$B=\left(K_{u \phi^{\circ}}^{*} U_{i 1}^{*} V_{j 2}^{*}+K_{d \phi^{\circ}}^{*} U_{i 2}^{*} V_{j 1}^{*}\right)$

$K_{u \phi^{\circ}}^{*}=\left(\cos \alpha, \sin \alpha, i \cos \beta_{\circ}, i \sin \beta_{\circ}\right)$

$K_{d \phi^{\circ}}^{*}=\left(-\sin \alpha, \cos \alpha, i \sin \beta_{\circ},-i \cos \beta_{\circ}\right)$

$\mathrm{U}$ and $\mathrm{V}$ are unitary matrices

\subsection{Calculation Cross Sections in (Pb) for Group (II):}

The cross sections as a function of center of mass energy for the Feynman diagrams of fig. (4) Calculated and the results given in fig.5 (a-d) by interchanging the mass of charginos $\left(\mathrm{m}_{\tilde{\chi}_{\mathrm{i}}^{-}}, \mathrm{m}_{\tilde{\chi}_{\mathrm{j}}^{+}}\right)$and the mass of Neutralino $\mathrm{m}_{\tilde{\chi}_{\ell}^{\circ}}$

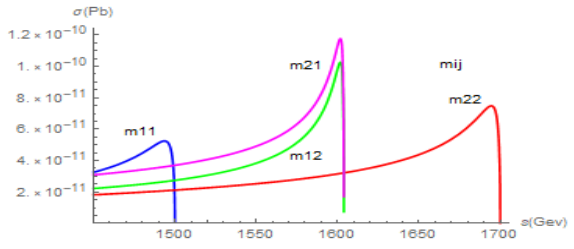

Fig. (5.a) $m_{\tilde{\chi}_{1}^{0}}=300 \mathrm{GeV}$

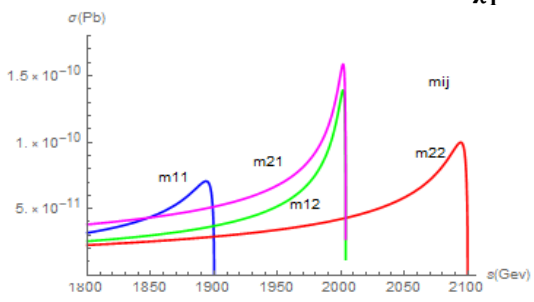

Fig. (5.c) $\mathbf{m}_{\tilde{\chi}_{3}^{0}}=700 \mathrm{GeV}$

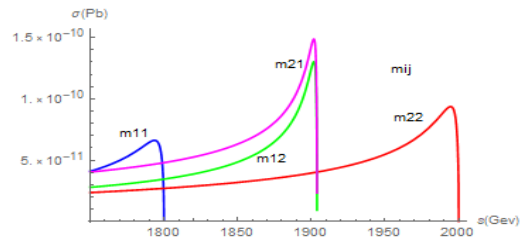

Fig. (5.b) $m_{\tilde{\chi}_{2}^{0}}=600 \mathrm{GeV}$

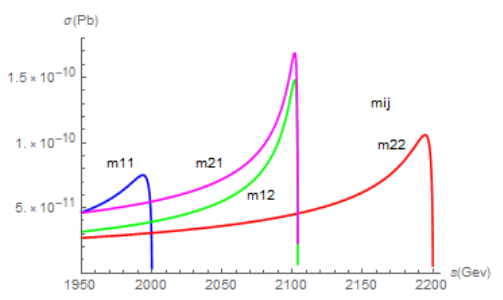

Fig. (5.d) $\mathbf{m}_{\tilde{\chi}_{4}^{0}}=800 \mathbf{~ G e V}$

Fig. 5 (a-d): The cross sections for the process $e^{-}\left(p_{1}\right)+e^{+}\left(p_{3}\right) \rightarrow \tilde{\chi}_{i}^{-}\left(p_{2}\right)+\tilde{\chi}_{j}^{+}\left(p_{4}\right)+\tilde{\chi}_{\ell}^{0}\left(p_{5}\right)$ as a function of center of mass energy via $\tilde{\chi}^{0}$ and $h^{o}$ propagators by interchanging the mass of charginos $\left(\mathrm{m}_{\tilde{\chi}_{i}^{-}}, \mathrm{m}_{\tilde{\chi}_{j}^{+}}\right)$at different mass of Neutralino $m_{\tilde{\chi}_{\rho}^{\circ}}$

\begin{tabular}{|c|c|c|c|c|c|c|c|c|}
\hline \multicolumn{9}{|c|}{$\mathbf{e}^{-}\left(\mathbf{P}_{\mathbf{1}}\right)+\mathbf{e}^{+}\left(\mathbf{P}_{3}\right) \rightarrow \mathbf{h}^{\mathbf{o}}\left(\mathbf{P}_{2}+\mathbf{P}_{\mathbf{4}}\right) \rightarrow \chi_{l}^{-}\left(\mathbf{P}_{2}\right)+\chi_{j}^{+}\left(\mathbf{P}_{\mathbf{4}}\right)$} \\
\hline \multirow{3}{*}{$\mathbf{m}_{\tilde{\chi}_{i}^{-}} \mathbf{m}_{\tilde{\chi}_{j}^{+}}$} & \multicolumn{2}{|l|}{$\tilde{\chi}_{1}^{0}=300$} & \multicolumn{2}{|c|}{$\widetilde{\chi}_{2}^{0}=600$} & \multicolumn{2}{|l|}{$\tilde{\chi}_{3}^{0}=700$} & \multicolumn{2}{|l|}{$\tilde{\chi}_{4}^{0}=\mathbf{8 0 0}$} \\
\hline & \multicolumn{2}{|c|}{ Fig. (3.a) } & \multicolumn{2}{|c|}{ Fig. (3.b) } & \multicolumn{2}{|c|}{ Fig. (3.c) } & \multicolumn{2}{|c|}{ Fig. (3.d) } \\
\hline & S(Gev) & $\sigma(\mathbf{P b})$ & S(Gev) & $\sigma(\mathbf{P b})$ & S(Gev) & $\sigma(\mathbf{P b})$ & S(Gev) & $\sigma(\mathbf{P b})$ \\
\hline 600,600 & 1492 & $\begin{array}{l}5.2 \\
\times 10^{-11}\end{array}$ & 1792 & $\begin{array}{l}6.6 \\
\times 10^{-11}\end{array}$ & 1892 & $7 \times 10^{-11}$ & 1992 & $\begin{array}{l}7.4 \\
\times 10^{-11}\end{array}$ \\
\hline 700,700 & 1692 & $\begin{array}{l}7.4 \\
\times 10^{-11}\end{array}$ & 1992 & $\begin{array}{l}9.2 \\
\times 10^{-11}\end{array}$ & 2092 & $1 \times 10^{-10}$ & 2192 & $1 \times 10^{-10}$ \\
\hline 600,700 & 1599 & $1 \times 10^{-10}$ & 1899 & $\begin{array}{l}1.2 \\
\times 10^{-10}\end{array}$ & 1999 & $\begin{array}{l}1.3 \\
\times 10^{-10}\end{array}$ & 2099 & $\begin{array}{l}1.4 \\
\times 10^{-10}\end{array}$ \\
\hline 700,600 & 1599 & $\begin{array}{l}1.1 \\
\times 10^{-10}\end{array}$ & 1899 & $\begin{array}{l}1.4 \\
\times 10^{-10}\end{array}$ & 1999 & $\begin{array}{l}1.5 \\
\times 10^{-10}\end{array}$ & 2099 & $\begin{array}{l}1.6 \\
\times 10^{-10}\end{array}$ \\
\hline
\end{tabular}

Table (2): cross sections for the process $\mathrm{e}^{-}\left(\mathrm{P}_{1}\right)+\mathrm{e}^{+}\left(\mathrm{P}_{3}\right) \rightarrow \mathrm{h}^{\mathrm{o}}\left(\mathrm{P}_{2}+\mathrm{P}_{4}\right) \rightarrow \chi_{l}^{-}\left(\mathrm{P}_{2}\right)+\chi_{j}^{+}\left(\mathrm{P}_{4}\right)$ via $\tilde{\chi}^{0}$ and $\mathrm{h}^{\mathrm{o}}$ propagators by interchanging the mass of charginos $\left(\mathrm{m}_{\tilde{\chi}_{\mathrm{i}}^{-}}, \mathrm{m}_{\tilde{\chi}_{\mathrm{j}}^{+}}\right)$at different mass of Neutralino $\mathrm{m}_{\tilde{\chi}_{\ell}^{\circ}}$

\subsection{Results for Group (II):}

After studding the Feynman rules and calculate the cross sections as a function of center of mass energy (S) for the process $e^{-}\left(p_{1}\right)+e^{+}\left(p_{3}\right) \rightarrow \tilde{\chi}_{i}^{-}\left(p_{2}\right)+\tilde{\chi}_{j}^{+}\left(p_{4}\right)+\tilde{\chi}_{\ell}^{\circ}\left(p_{5}\right)$ via $\tilde{\chi}^{0}$ and $\mathrm{h}^{\mathrm{o}}$ propagators in fig.5 (a-d) we found that: At $\mathrm{S}$ increase from 1450 to 2200 we have different maximum values from the cross-sections at different values of Chargino mass $\left(\mathrm{m}_{\tilde{\chi}_{i}^{-}}, \mathrm{m}_{\tilde{\chi}_{j}^{+}}\right)$and different value of neutralino mass $\mathrm{m}_{\tilde{\chi}_{\ell}^{0}}$. From table (2) the best value 
of $\sigma$ is $\left(1.6 \times 10^{-10}\right) \mathrm{Pb}$ when masses of Charginos are $\mathrm{m}_{\chi_{i}^{-}}=700 \mathrm{GeV}, \mathrm{m}_{\chi_{j}^{+}}=600 \mathrm{GeV}$ and $\mathrm{m}_{\tilde{\chi}_{\ell}^{0}}=800 \mathrm{GeV}$

5. Feynman Diagram and M-Matrix for the process, when $\tilde{\chi}^{0}$ and $\mathrm{H}^{\circ}$ are the propagators Group (III):

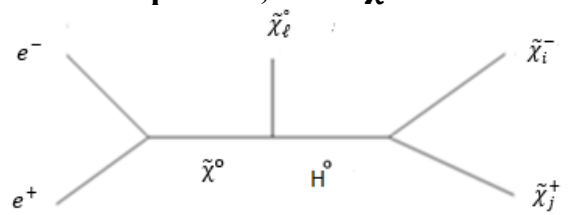

Fig. (6): Feynman diagram for the process $e^{-}\left(p_{1}\right)+e^{+}\left(p_{3}\right) \rightarrow \tilde{\chi}_{i}^{-}\left(p_{2}\right)+\tilde{\chi}_{j}^{+}\left(p_{4}\right)+\tilde{\chi}_{\ell}^{\circ}\left(p_{5}\right)$ via $\tilde{\chi}^{\mathrm{o}}$ and $\mathrm{H}^{\mathrm{o}}$ there are (129-192) diagrams.

- For group (III) there are 64 situations from $(129$ - 192) and the Matrix element is:

$$
M_{(129-192)}=\frac{-i g^{2} m_{e}}{4 \sqrt{2} m_{w} \sin \theta_{w}} \mathrm{U}_{\mathrm{e}^{-}}\left(\mathrm{P}_{1}\right) \overline{\mathrm{V}}_{\mathrm{e}^{+}}\left(\mathrm{P}_{3}\right) \gamma_{5} \frac{s+\mathrm{m}_{\chi}}{s^{2}-m_{\chi}^{2}} \overline{\mathrm{U}}_{\tilde{\chi}^{0}}\left(\mathrm{P}_{5}\right) \mathrm{C}\left(\sigma^{2}-\mathrm{m}_{\mathrm{H}}^{2}\right)^{-1} B \overline{\mathrm{U}}_{\tilde{\chi}^{-}}\left(\mathrm{P}_{2}\right) \overline{\mathrm{U}}_{\tilde{\chi}^{+}}\left(\mathrm{P}_{4}\right)
$$

\subsection{Calculation Cross Sections in (Pb) for Group (III):}

The Cross sections as a function of center of mass energy for the Feynman diagrams of fig. (6) Calculated and the results given in fig.7 (a-d) by interchanging the mass of charginos $\left(\mathrm{m}_{\tilde{\chi}_{\mathrm{i}}^{-}}, \mathrm{m}_{\tilde{\chi}_{\mathrm{j}}^{+}}\right)$and the mass of Neutralino $\mathrm{m}_{\tilde{\chi}_{\ell}}$

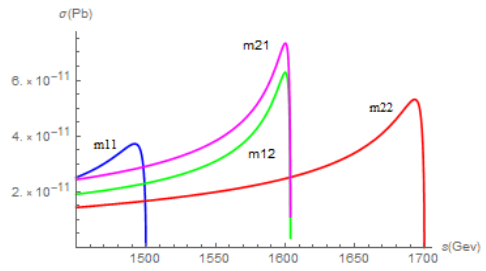

Fig. (7.a) $m_{\tilde{\chi}_{1}^{0}}=300 \mathrm{GeV}$

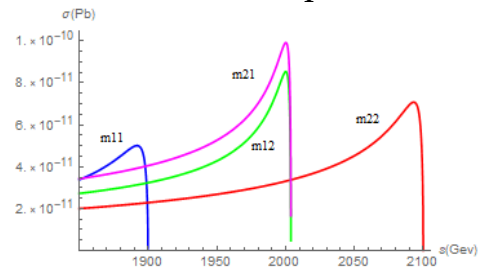

Fig. (7.c) $m_{\tilde{\chi}_{3}^{0}}=700 \mathrm{GeV}$

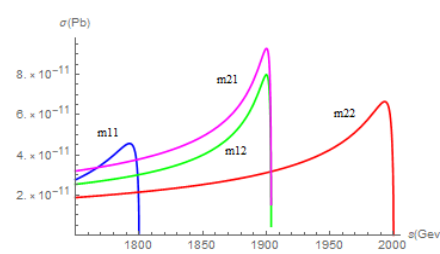

Fig. (7.b) $m_{\tilde{\chi}_{2}^{0}}=600 \mathrm{GeV}$

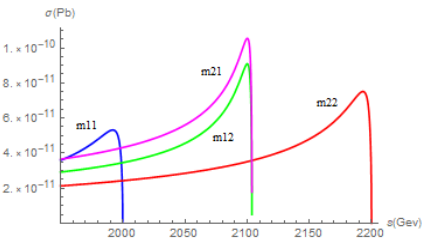

Fig. (7.d) $\mathbf{m}_{\tilde{\chi}_{\mathbf{4}}^{0}}=800 \mathbf{~ G e V}$

Fig. 7 (a-d): The cross sections for the process $e^{-}\left(p_{1}\right)+e^{+}\left(p_{3}\right) \rightarrow \tilde{\chi}_{i}^{-}\left(p_{2}\right)+\tilde{\chi}_{j}^{+}\left(p_{4}\right)+\tilde{\chi}_{\ell}^{\circ}\left(p_{5}\right)$ as a function of center of mass energy via $\tilde{\chi}^{\mathrm{o}}$ and $\mathrm{H}^{\mathrm{o}}$ propagators by interchanging the mass of charginos $\left(\mathrm{m}_{\tilde{\chi}_{i}^{-}}, \mathrm{m}_{\tilde{\chi}_{j}^{+}}\right)$at different mass of Neutralino $\mathrm{m}_{\tilde{\chi}_{\ell}^{\circ}}$.

Table (3): cross sections for the process $\mathrm{e}^{-}\left(\mathrm{P}_{1}\right)+\mathrm{e}^{+}\left(\mathrm{P}_{3}\right) \rightarrow \mathrm{H}^{\mathrm{o}}\left(\mathrm{P}_{2}+\mathrm{P}_{4}\right) \rightarrow \chi_{l}^{-}\left(\mathrm{P}_{2}\right)+\chi_{j}^{+}\left(\mathrm{P}_{4}\right)$ via $\tilde{\chi}^{\mathrm{o}}$ and $\mathrm{H}^{\mathrm{o}}$ Propagators by interchanging the mass of charginos $\left(\mathrm{m}_{\tilde{\chi}_{\mathrm{i}}^{-}}, \mathrm{m}_{\tilde{\chi}_{\mathrm{j}}^{+}}\right)$at different mass of Neutralino $\mathrm{m}_{\tilde{\chi}_{\ell}}$

\begin{tabular}{|c|c|c|c|c|c|c|c|c|}
\hline \multicolumn{9}{|c|}{$\mathbf{e}^{-}\left(\mathbf{P}_{1}\right)+\mathbf{e}^{+}\left(\mathbf{P}_{3}\right) \rightarrow \mathbf{H}^{\mathbf{0}}\left(\mathbf{P}_{2}+\mathbf{P}_{4}\right) \rightarrow \chi_{l}^{-}\left(\mathbf{P}_{2}\right)+\chi_{j}^{+}\left(\mathbf{P}_{4}\right)$} \\
\hline \multirow{3}{*}{$\mathbf{m}_{\tilde{\chi}_{\mathbf{i}}^{-}}^{-\mathbf{m}_{\tilde{\chi}_{j}^{+}}^{+}}$} & \multicolumn{2}{|l|}{$\widetilde{\chi}_{1}^{0}=\mathbf{3 0 0}$} & \multicolumn{2}{|l|}{$\widetilde{\chi}_{2}^{0}=600$} & \multicolumn{2}{|c|}{$\tilde{\chi}_{3}^{0}=700$} & \multicolumn{2}{|l|}{$\tilde{\chi}_{4}^{0}=\mathbf{8 0 0}$} \\
\hline & \multicolumn{2}{|c|}{ Fig. (3.a) } & \multicolumn{2}{|c|}{ Fig. (3.b) } & \multicolumn{2}{|c|}{ Fig. (3.c) } & \multicolumn{2}{|c|}{ Fig. (3.d) } \\
\hline & S(Gev) & $\sigma(\mathbf{P b})$ & S(Gev) & $\sigma(\mathbf{P b})$ & S(Gev) & $\sigma(\mathbf{P b})$ & S(Gev) & $\sigma(\mathbf{P b})$ \\
\hline 600,600 & 1491 & $\begin{array}{l}3.7 \\
\times 10^{-11}\end{array}$ & 1792 & $\begin{array}{l}4.5 \\
\times 10^{-11}\end{array}$ & 1891 & $5 \times 10^{-11}$ & 1991 & $5.3 \times 10^{-11}$ \\
\hline 700,700 & 1693 & $\begin{array}{l}5.3 \\
\times 10^{-11}\end{array}$ & 1992 & $\begin{array}{l}6.6 \\
\times 10^{-11}\end{array}$ & 2092 & $7 \times 10^{-11}$ & 2192 & $7.5 \times 10^{-11}$ \\
\hline 600,700 & 1598 & $6 \times 10^{-11}$ & 1899 & $\begin{array}{l}7.9 \\
\times 10^{-11}\end{array}$ & 1999 & $\begin{array}{l}8.5 \\
\times 10^{-11}\end{array}$ & 2099 & $9 \times 10^{-11}$ \\
\hline 700,600 & 1598 & $7 \times 10^{-11}$ & 1899 & $\begin{array}{l}9.2 \\
\times 10^{-11}\end{array}$ & 1999 & $\begin{array}{l}9.9 \\
\times 10^{-11}\end{array}$ & 2099 & $1 \times 10^{-10}$ \\
\hline
\end{tabular}

5.2 Results for Group (III):

After studding the Feynman rules and calculate the cross sections as a function of center of mass energy (S) for 
the process $e^{-}\left(p_{1}\right)+e^{+}\left(p_{3}\right) \rightarrow \tilde{\chi}_{i}^{-}\left(p_{2}\right)+\tilde{\chi}_{j}^{+}\left(p_{4}\right)+\tilde{\chi}_{\ell}^{\circ}\left(p_{5}\right)$ via $\tilde{\chi}^{\mathrm{o}}$ and $\mathrm{H}^{\mathrm{o}}$ propagators in fig.7 (a-d) we found that: At $\mathrm{S}$ increase from 1450 to 2200 we have different maximum values from the cross-sections at different values of Chargino mass $\left(\mathrm{m}_{\chi_{i}^{-}}, \mathrm{m}_{\chi_{j}^{+}}\right)$and different value of neutralino mass $\mathrm{m}_{\tilde{\chi}_{\ell}^{0}}$. From table (3) the best value of $\sigma$ is $\left(1 \times 10^{-10}\right) \mathrm{Pb}$ when masses of Charginos are $\mathrm{m}_{\chi_{i}^{-}}=700 \mathrm{GeV}, \mathrm{m}_{\chi_{j}^{+}}=600 \mathrm{GeV}$ and $\mathrm{m}_{\tilde{\chi}_{\ell}^{0}}=800 \mathrm{GeV}$

\section{Discussion}

Figs. $(3,5,7)$. Shows the cross-sections calculation for the process $\mathrm{e}^{-}\left(\mathrm{P}_{1}\right)+\mathrm{e}^{+}\left(\mathrm{P}_{3}\right) \rightarrow \tilde{\chi}_{i}^{-}\left(\mathrm{P}_{2}\right)+\tilde{\chi}_{j}^{+}\left(\mathrm{P}_{4}\right)+$ $\tilde{\chi}_{\ell}^{0}\left(\mathrm{P}_{5}\right)$ as a function of center of mass energy $\mathrm{S}$, via $\tilde{\chi}^{\circ}$ and $\mathrm{Z}^{\mathrm{o}}$ boson propagators group (I), via $\tilde{\chi}^{\circ}$ and $\mathrm{h}^{\mathrm{o}}$ boson propagators group (II) and via $\tilde{\chi}^{\circ}$ and $\mathrm{H}^{\circ}$ boson propagators (III) respectively. If center of mass energy $\mathrm{S}$ increases the cross-sections increase, but after certain value of $\mathrm{S}$ the value of cross sections decrease and the range of center of mass energy from (1000-2100)

The following table shows that the peak values of the cross section $\sigma(\mathrm{Pb})$ for each mode and the corresponding center of mass energy $\mathrm{S}(\mathrm{GeV})$ at different masses of neutralino $\tilde{\chi}_{\ell}^{0}$ and different masses of charginos $\tilde{\chi}_{i}^{-}, \tilde{\chi}_{j}^{+}$to determine the best value of cross-section for the reaction $\mathrm{e}^{-}\left(\mathrm{P}_{1}\right)+\mathrm{e}^{+}\left(\mathrm{P}_{3}\right) \rightarrow \tilde{\chi}_{i}^{-}\left(\mathrm{P}_{2}\right)+$ $\tilde{\chi}_{j}^{+}\left(\mathrm{P}_{4}\right)+\tilde{\chi}_{\ell}^{0}\left(\mathrm{P}_{5}\right)$

Table (4): The peak values of the cross sections of the interaction $\mathrm{e}^{-}\left(\mathrm{P}_{1}\right)+\mathrm{e}^{+}\left(\mathrm{P}_{3}\right) \rightarrow \tilde{\chi}_{i}^{-}\left(\mathrm{P}_{2}\right)+\tilde{\chi}_{j}^{+}\left(\mathrm{P}_{4}\right)+\tilde{\chi}_{\ell}^{0}\left(\mathrm{P}_{5}\right)$, with different masses of Charginos $\left(\mathrm{m}_{\chi_{i}^{-}}, \mathrm{m}_{\chi_{j}^{+}}\right)$and neutralino $\mathrm{m}_{\tilde{\chi}_{\ell}^{\circ}}$ at different values of incident energies.

\begin{tabular}{|c|c|c|c|c|c|c|}
\hline Group.no. & $\begin{array}{l}\mathbf{e}^{-}\left(\mathbf{P}_{\mathbf{1}}\right)+\mathbf{e}^{+}\left(\mathbf{P}_{3}\right) \rightarrow \chi_{\mathbf{i}}^{-}\left(\mathbf{P}_{2}\right)+ \\
\chi_{j}^{+}\left(\mathbf{P}_{\mathbf{4}}\right)+\tilde{\chi}_{\boldsymbol{\ell}}^{0}\left(\mathbf{P}_{\mathbf{5}}\right)\end{array}$ & Fig. no. & $\boldsymbol{m}_{\widetilde{\chi}_{\ell}^{0}}$ & $\mathbf{m}_{\tilde{\chi}_{\mathbf{i}}^{-}} \mathbf{m}_{\tilde{\chi}_{\mathbf{j}}^{+}}$ & $\begin{array}{l}\mathrm{S}(\mathrm{GeV}) \\
\text { at } \max \sigma\end{array}$ & $\sigma(\mathrm{Pb})$ \\
\hline \multirow[t]{4}{*}{$\mathbf{I}$} & \multirow{4}{*}{ Production via $\tilde{\chi}^{0}$ and $\mathrm{Z}^{0}$} & $3 . \mathrm{a}$ & $300 \mathrm{GeV}$ & 700,700 & 1202 & 0.030 \\
\hline & & $3 . \mathrm{b}$ & $600 \mathrm{GeV}$ & 700,700 & 1350 & 0.053 \\
\hline & & 3.c & $700 \mathrm{GeV}$ & 700,700 & 1041 & 0.063 \\
\hline & & $3 . d$ & $800 \mathrm{GeV}$ & 700,700 & 1015 & 0.072 \\
\hline \multirow[t]{4}{*}{ II } & \multirow[t]{4}{*}{ Production via $\tilde{\chi}^{0}$ and $h^{0}$} & $5 . \mathrm{a}$ & $300 \mathrm{GeV}$ & 700,600 & 1599 & $\begin{array}{l}1.1 \\
\times 10^{-10} \\
\end{array}$ \\
\hline & & $5 . b$ & $600 \mathrm{GeV}$ & 700,600 & 1899 & $\begin{array}{l}1.4 \\
\times 10^{-10} \\
\end{array}$ \\
\hline & & 5.c & $700 \mathrm{GeV}$ & 700,600 & 1999 & $\begin{array}{l}1.5 \\
\times 10^{-10} \\
\end{array}$ \\
\hline & & 5.d & $800 \mathrm{GeV}$ & 700,600 & 2099 & $\begin{array}{l}1.6 \\
\times 10^{-10}\end{array}$ \\
\hline \multirow[t]{4}{*}{ III } & \multirow[t]{4}{*}{ Production via $\tilde{\chi}^{0}$ and $\mathrm{H}^{0}$} & $7 . \mathrm{a}$ & $300 \mathrm{GeV}$ & 700,600 & 1598 & $7 \times 10^{-11}$ \\
\hline & & $7 . b$ & $600 \mathrm{GeV}$ & 700,600 & 1899 & $\begin{array}{l}9.2 \\
\times 10^{-11}\end{array}$ \\
\hline & & $7 . \mathrm{c}$ & $700 \mathrm{GeV}$ & 700,600 & 1999 & $\begin{array}{l}9.9 \\
\times 10^{-11} \\
\end{array}$ \\
\hline & & $7 . d$ & $800 \mathrm{GeV}$ & 700,600 & 2099 & $\begin{array}{l}1.1 \\
\times 10^{-10}\end{array}$ \\
\hline
\end{tabular}

\section{Conclusion}

From table (4), we have success to identify the scenario for highest cross section for the reactione ${ }^{-}\left(\mathrm{P}_{1}\right)+\mathrm{e}^{+}\left(\mathrm{P}_{3}\right) \rightarrow$ $\tilde{\chi}_{i}^{-}\left(\mathrm{P}_{2}\right)+\tilde{\chi}_{j}^{+}\left(\mathrm{P}_{4}\right)+\tilde{\chi}_{l}^{0}\left(\mathrm{P}_{5}\right)$.

-The best cross section goes up to $(0.072 \mathrm{~Pb})$ at $(\mathrm{S}=1015 \mathrm{GeV})$ when masses of Charginos are $\mathrm{m}_{\widetilde{\chi}_{i}^{-}}=700 \mathrm{GeV}$, $\mathrm{m}_{\tilde{\chi}_{j}^{+}}=700 \mathrm{GeV}$ and $\mathrm{m}_{\tilde{\chi}_{\ell}^{0}}=800 \mathrm{GeV}$, via $\tilde{\chi}^{0}$ and $Z^{0}$ boson propagators exchange in Fig. (3.d) for the reaction $\mathrm{e}^{-}\left(\mathrm{P}_{1}\right)+\mathrm{e}^{+}\left(\mathrm{P}_{3}\right) \rightarrow Z^{0}\left(P_{2}+P_{4}\right) \rightarrow \tilde{\chi}_{i}^{-}\left(\mathrm{P}_{2}\right)+\tilde{\chi}_{j}^{+}\left(\mathrm{P}_{4}\right)$ in group $(\mathrm{I})$

- The competing cross section goes up to $\left(1.6 \times 10^{-10} \mathrm{~Pb}\right)$ at $(\mathrm{S}=2099 \mathrm{GeV})$ when masses of Charginos are $\mathrm{m}_{\tilde{\chi}_{i}^{-}}=700 \mathrm{GeV}, \mathrm{m}_{\tilde{\chi}_{j}^{+}}=600 \mathrm{GeV}$ and $\mathrm{m}_{\tilde{\chi}_{\ell}^{0}}=800 \mathrm{GeV}$, via $\tilde{\chi}^{0}$ and $h^{0}$ boson propagators exchange in Fig. (5.d) for the reaction $\mathrm{e}^{-}\left(\mathrm{P}_{1}\right)+\mathrm{e}^{+}\left(\mathrm{P}_{3}\right) \rightarrow h^{0}\left(P_{2}+P_{4}\right) \rightarrow \tilde{\chi}_{i}^{-}\left(\mathrm{P}_{2}\right)+\tilde{\chi}_{j}^{+}\left(\mathrm{P}_{4}\right)$ in group (II)

-the other cross section is $\left(1.1 \times 10^{-10} \mathrm{~Pb}\right)$ at $(\mathrm{S}=2099 \mathrm{GeV})$ when masses of Charginos are $\mathrm{m}_{\tilde{\chi}_{i}^{-}}=700 \mathrm{GeV}$, $\mathrm{m}_{\tilde{\chi}_{j}^{+}}=600 \mathrm{GeV}$ and $\mathrm{m}_{\tilde{\chi}_{\ell}^{0}}=800 \mathrm{GeV}$, via $\tilde{\chi}^{0}$ and $H^{0}$ boson propagators exchange in Fig. (7.d) for the reaction $\mathrm{e}^{-}\left(\mathrm{P}_{1}\right)+\mathrm{e}^{+}\left(\mathrm{P}_{3}\right) \rightarrow H^{0}\left(P_{2}+P_{4}\right) \rightarrow \tilde{\chi}_{i}^{-}\left(\mathrm{P}_{2}\right)+\tilde{\chi}_{j}^{+}\left(\mathrm{P}_{4}\right)$ in group (III) 


\section{References}

[1] GODBOLE, Rohini M. Higgs and SUSY searches at future colliders. Pramana, (2000), 54.4: 499-518.

[2] DEV, PS Bhupal, et al. Determining the CP Properties of the Higgs Boson. Physical review letters, (2008), 100.5: 051801

[3] IGO-KEMENES, P. Neutral Higgs bosons in the standard model and in the minimal supersymmetric model: Searches at LEP. Pramana, (2004), 62.3: 555-560.

[4] M.M. Ahmed, Asmaa, A. A.; Zainab, A. H. Production of Neutralino and Tow Higss Bosons from electronpositron annihilation via $H^{\circ}$ and $Z^{\circ}$ propagators. Journal of Modern Trends in Physics Research, (2019), 19: 17-24.

[5] COLLABORATION, C. M. S. Search for a low-mass pseudoscalar Higgs boson produced in association with a bb pair in pp collisions at. Physics Letters B, (2016), 758: 296-320.

[6] Herrero, Maria J. "The Higgs system in and beyond the standard model." Lectures on Particle Physics, Astrophysics and Cosmology. Springer, Cham, (2015). 188-252.

[7] DOVA, María Teresa; FERRARI, Sergio. On the determination of CP-even and CP-odd components of a mixed CP Higgs boson at e+ e- linear colliders. Physics Letters B, (2005), 605.3-4: 376-383.

[8] Manimala C, Sven H, Ipsita S. Improved (g-2) $\mu$ measurements and supersymmetry. The European Physical Journal. C, Particles and Fields. (2020);80(10).

[9] SASAKI, Ken; UEMATSU, Tsuneo. CP-odd Higgs boson production in e $\gamma$ collisions. Physics Letters B, (2018), 781: 290-294.

[10] MAHMOUD, Ibrahim S.; A EL-AZIM, T. Production of Lightest Neutral MSSM Higgs Boson in Association with Neutralinos at Electron Positron Colliders. Arab Journal of Nuclear Sciences and Applications, (2018), 51.2: $42-50$.

[11] J.F.Gunion and H.E, Haber Nucl.Phys.B ,(1986),272.1 ; J.F. Gunion, H.E. Haber, Nucl. Phys. B, (1986) 278 .449; J.F.Gunion and H.E, Haber.G.I Kane and S.Dawson, Nucl.Phys. B (1990) 84.

[12] GLADYSHEV, A. V.; KAZAKOV, D. I. Is (Low Energy) SUSY still alive? arXiv preprint arXiv:1212.2548, (2012).

[13] H.E.Haber and G.L.Kane. (1986) Nucl.Phys.B 272 ,1. GUNION, John F.; HABER, Howard E. Higgs bosons in supersymmetric models (I). Nuclear Physics B, (1986), 272.1: 1-76.

[14] Nous MH, El-Kishen M, El-Azem TA. Production cross sections for the multi-Higgs-boson process $e^{+} e^{-} \rightarrow H_{i}^{\circ} H^{+} H^{-}$. Physical Review D. (1991);44(11):3427

[15] ATHRON, Peter, et al. Combined collider constraints on neutralinos and charginos. The European Physical Journal C, (2019), 79.5: 1-52.

[16] Aad G, Abbott B, Abbott DC, Abud AA, Abeling K, Abhayasinghe DK, Abidi SH, AbouZeid OS, Abraham NL, Abramowicz H, Abreu H. Search for direct production of electroweakinos in final states with missing transverse momentum and a Higgs boson decaying into photons in pp collisions at $\sqrt{\mathrm{s}}=13 \mathrm{TeV}$ with the ATLAS detector. Journal of High Energy Physics;(2020) (10):1-46

[17] Aldufeery E, Binjonaid M. Dark matter constraints and the neutralino sector of the scNMSSM. Universe. $(2021) ; 7(2): 31$

[18] MARTIN, Stephen P. A supersymmetry primer. In: Perspectives on supersymmetry. (1998). p. 1-98.

[19] BERTONE, Gianfranco (ed.). Particle dark matter: observations, models and searches. Cambridge University Press, (2010).

[20] HEUER, R.-D., et al. TESLA technical design report part III: Physics at an e+ e-linear collider. arXiv preprint hep-ph/0106315, (2001).

[21] de Vera MT, Berggren M, List J. Chargino production at the ILC. arXiv preprint arXiv:2002.01239. (2020)

[22] AAD, Georges, et al. Search for charginos nearly mass degenerate with the lightest neutralino based on a disappearing-track signature in $\mathrm{p} p$ collisions at $(\mathrm{s})=8 \mathrm{TeV}$ with the ATLAS detector. Physical Review D, (2013), 88.11: 112006.

[23] CHOI, S. Y., et al. Chargino pair production in $e^{-} e^{+}$collisions. The European Physical Journal C-Particles and Fields, (1999), 7.1: 123-134.

[24] H.Baer, V.Barger and H.Serce (2019) [hep-ph/1907.06693v1].

[25] LI, Tianjun. Determining $M_{1}, M_{2} \mu$ and $\tan \beta$ in MSSM from Chargino and Neutralino Masses. (1998).

[26] S.M.Bilenky and J.Hosek, (1990) Phys.Rep.,90,73.

[27] WILLIAMSON JR, W. Pedestrian Covariant Phase-Space Integration. American Journal of Physics, (1965), 33.12: 987-994.

[28] ALLANACH, Benjamin C., et al. SUSY parameter analysis at TeV and Planck scales. Nuclear Physics, B, (2004), 135, 107

[29] K.Desch, J.Kalinowski,G.Moortgat-Pick, M.M.Nojiri and G.Rolesello, JHEP 0402(2004)035 [hep$\mathrm{ph} / 032069]$. 